\title{
Sequential measurement-driven multi-target Bayesian filter
}

\author{
Zong-xiang Liu*, Li-juan Li, Wei-xin Xie and Liang-qun Li
}

\begin{abstract}
Bayesian filter is an efficient approach for multi-target tracking in the presence of clutter. Recently, considerable attention has been focused on probability hypothesis density (PHD) filter, which is an intensity approximation of the multi-target Bayesian filter. However, PHD filter is inapplicable to cases in which target detection probability is low. The use of this filter may result in a delay in data processing because it handles received measurements periodically, once every sampling period. To track multiple targets in the case of low detection probability and to handle received measurements in real time, we propose a sequential measurement-driven Bayesian filter. The proposed filter jointly propagates the marginal distributions and existence probabilities of each target in the filter recursion. We also present an implementation of the proposed filter for linear Gaussian models. Simulation results demonstrate that the proposed filter can more accurately track multiple targets than the Gaussian mixture PHD filter or cardinalized PHD filter.
\end{abstract}

Keywords: Multi-target tracking; Bayesian filter; Probability hypothesis density filter; Marginal distribution; Existence probability

\section{Introduction}

Multi-target tracking aims to detect individual targets in the surveillance region of interest and estimate their states according to a sequence of noisy and cluttered measurements collected by sensors. The most efficient technique for multi-target tracking is the multi-target Bayesian filter, which propagates joint posterior distribution of the multi-target state $[1,2]$. However, such propagation is computationally intensive because of the high dimensionality of the multi-target state space $[2,3]$. With the use of the Bayesian framework to propagate the posterior intensity of multiple targets recursively, the probability hypothesis density (PHD) filter provides a numerically tractable solution to this problem [2, 3]. Two numerical solutions, namely sequential Monte Carlo (SMC) [4-9] and Gaussian mixtures (GM) [10-17], have been developed for the PHD filter. Extensions of the PHD filter have also been proposed to improve its performance. PHD filters with observation-driven birth intensity were independently proposed in $[16,18,19]$ to eliminate the need for exact knowledge of birth

\footnotetext{
* Correspondence: liuzx@szu.edu.cn

ATR Key Laboratory, College of Information and Engineering, Shenzhen University, P.O. Box 2-603, 518060 Shenzhen, China
}

intensity. Methods for maintaining track continuity were proposed in $[4,20]$ for the SMC-PHD filter and in [21] for the GM-PHD filter. To improve the accuracy and stability of the target number estimate, the cardinalized PHD (CPHD) filter, which jointly propagates moment and cardinality, was proposed in [22]. Methods for estimating an unknown clutter rate, which is an important parameter of the PHD and CPHD filters, were proposed in [23] and [24]. In [12], the GM-PHD filter was extended to linear jump Markov multi-target models for use in tracking maneuvering targets.

Although the PHD filter has several advantages, it becomes inefficient in cases with low target detection probability. This inefficiency occurs because the PHD filter has a weak memory and is easily influenced by new incoming measurements $[2,17,22]$. Owing to its weak memory, the PHD filter fails to provide state estimates of existing targets if these targets are missing from new incoming measurements [2]. Moreover, the PHD filter may result in data processing delay. This delay occurs because the PHD filter handles new incoming measurements periodically, once every sampling period. In this manner, new measurements have to be gathered for a sampling period before being processed. Therefore, a

\section{穴 Springer}


significant delay arises from a long sampling period. The CPHD filter has a better memory than the PHD filter but has a slower response in detecting target appearance and disappearance and is less influenced by new incoming measurements. Despite its strong memory, the CPHD filter may also be inefficient in cases with low detection probability because it has more difficulty than the PHD filter in giving state estimates of new targets because of its slow response to new targets. In addition, similar to the PHD filter, the CPHD filter may also result in data processing delay because it also handles new incoming measurements periodically.

To resolve the multi-target tracking problem efficiently in the case of low detection probability and to reduce data processing delay, we propose a sequential measurement-driven Bayesian filter. This filter propagates the marginal distributions and existence probabilities of each target in the filter recursion and uses received measurements to generate new marginal distributions and update existing marginal distributions. In this filter, we use the prediction marginal distribution and existence probability of a target as its update marginal distribution and existence probability, respectively, and propagate them in the filter recursion if this target is missing from the incoming measurements. Thus, the proposed filter has a sufficient memory to missing targets, which enables this filter applicable to tracking multiple targets in the case of low detection probability. Moreover, this filter reduces the data processing delay that exists in PHD and CPHD filters because new incoming measurements can be processed whenever they become available. We also propose a closed-form solution and implementation of the proposed filter for linear Gaussian models. In terms of optimal sub-pattern assignment (OSPA) distance [25], we compare the proposed filter with the PHD and CPHD filters through simulation. These three filters are capable of tracking multiple targets in the presence of clutter as well as target appearance and disappearance. Among the three filters, the proposed filter is the best at tracking multiple targets in cases of low detection probability.

The main contributions of this paper are twofold. First, we propose a new Bayesian filter for sequentially handling new measurements. The proposed filter handles received measurements in real time and is applicable to tracking multiple targets in the case of low detection probability. Second, we present a novel implementation of the proposed filter for linear Gaussian models.

The remainder of this paper is organized as follows: Section 2 briefly introduces the multi-target Bayesian filter. Section 3 proposes the sequential measurementdriven multi-target Bayesian filter to propagate marginal distribution. Section 4 discusses the implementation of the proposed filter in linear Gaussian models. Section 5 evaluates the performance of the proposed filter. Section 6 states the drawn conclusions.

\section{Multi-target Bayesian filter}

We first provide a brief description of the multi-target Bayesian filter [1]. In a multi-target Bayesian filter, the distribution of interest is the joint posterior $f\left(\mathbf{x}_{k} \mid \mathbf{y}_{1: k}\right)$, which is also known as the filtering distribution, where $k$ denotes the time step, $\mathbf{x}_{k}=\left(\mathbf{x}_{1, k} \cdots \mathbf{x}_{N, k}\right)$ is the multitarget state at time step $k, N$ is the target number, and $\mathbf{y}_{1: k}=\left(\mathbf{y}_{1} \cdots \mathbf{y}_{k}\right)$ represents all observations from time step 1 to time step $k$. The filtering distribution of a multi-target Bayesian filter can be computed by using a two-step recursion.

Prediction step:

$$
f\left(\mathbf{x}_{k} \mid \mathbf{y}_{1: k-1}\right)=\int f\left(\mathbf{x}_{k} \mid \mathbf{x}_{k-1}\right) f\left(\mathbf{x}_{k-1} \mid \mathbf{y}_{1: k-1}\right) d \mathbf{x}_{k-1}
$$

Update step:

$$
f\left(\mathbf{x}_{k} \mid \mathbf{y}_{1: k}\right)=\frac{g\left(\mathbf{y}_{k} \mid \mathbf{x}_{k}\right) f\left(\mathbf{x}_{k} \mid \mathbf{y}_{1: k-1}\right)}{f\left(\mathbf{y}_{k} \mid \mathbf{y}_{1: k-1}\right)}
$$

where $f\left(\mathbf{x}_{k} \mid \mathbf{x}_{k}-1\right)$ denotes the Markov transition probability from state $\mathbf{x}_{k-1}$ at time step $k-1$ to state $\mathbf{x}_{k}$ at time step $k, g\left(\mathbf{y}_{k} \mid \mathbf{x}_{k}\right)$ is the probability density that state $\mathbf{x}_{k}$ at time step $k$ generates measurement $\mathbf{y}_{k}$ at time step $k$, and $f\left(\mathbf{y}_{k} \mid \mathbf{y}_{1: k-1}\right)$ is given by:

$$
f\left(\mathbf{y}_{k} \mid \mathbf{y}_{1: k-1}\right)=\int g\left(\mathbf{y}_{k} \mid \mathbf{x}_{k}\right) f\left(\mathbf{x}_{k} \mid \mathbf{y}_{1: k-1}\right) d \mathbf{x}_{k}
$$

Equation 2 clearly shows that the new filtering distribution is obtained by directly applying the Bayesian rule. The generally intractable multi-target Bayesian filter involves integrals of high dimensions. This intractability is usually resolved by applying fixed grid approximation, SMC approximation, and PHD approximation [1, 2].

\section{Sequential measurement-driven multi-target Bayesian filter}

Similar to the PHD filter in [10] and CPHD filter in [22], we consider the following assumptions to present the sequential measurement-driven Bayesian (SMB) filter.

\section{A1. Targets evolve and generate observations independent of one another. \\ A2. Clutter is independent of target-originated measurements. \\ A3. The survival and detection probabilities of each target are state independent.}

To derive the SMB filter conveniently, we also assume that the number of targets at time step $k-1$ is $N_{k-1}$; the states of individual targets at time step $k-1$ are $\mathbf{x}_{i, k-1}$, 
$i=1 \cdots N_{k-1}$; the marginal distributions and existence probabilities of individual targets at time step $k-1$ are $f_{i}\left(\mathbf{x}_{i, k-1} \mid \mathbf{y}_{1: k-1}\right), i=1 \cdots N_{k-1}$ and $p_{\mathrm{i}, k-1}, i=1 \cdots$ $N_{k-1}$, respectively; and $\mathbf{y}_{1: k-1}=\left(\mathbf{y}_{1} \cdots \mathbf{y}_{k-1}\right)$ represents all observations up to time step $k-1$. The objective is to determine the marginal distributions for each target and their existing probabilities at time step $k$.

Individually using Equation 1 to process each marginal distribution $f_{i}\left(\mathbf{x}_{i, k}-1 \mid \mathbf{y}_{1}: k-1\right)$ at time step $k-1$, we obtain individual prediction distributions $f_{i}\left(\mathbf{x}_{i, k} \mid \mathbf{y}_{1: k-1}\right)$, $i=1 \cdots N_{k-1}$ at time step $k$ as:

$$
\begin{aligned}
f_{i}\left(\mathbf{x}_{i, k} \mid \mathbf{y}_{1: k-1}\right) & =\int f_{i}\left(\mathbf{x}_{i, k} \mid \mathbf{x}_{i, k-1}\right) f_{i}\left(\mathbf{x}_{i, k-1} \mid \mathbf{y}_{1: k-1}\right) d \mathbf{x}_{i, k-1} \\
i & =1 \cdots N_{k-1}
\end{aligned}
$$

The existence probability $p_{i, k \mid k}-1$ of the prediction distribution $f_{i}\left(\mathbf{x}_{i, k} \mid \mathbf{y}_{1: k-1}\right)$ is given by:

$$
p_{i, k \mid k-1}=p_{S, k}\left(t_{k}, t_{k-1}\right) p_{i, k-1}, i=1 \cdots N_{k-1}
$$

where $p_{S, k}\left(t_{k}, t_{k}-1\right)$ is the time-dependent survival probability of the target, and $t_{k}$ is the time of time step $k$.

Assuming that the observations at time step $k$ are denoted by $\mathbf{y}_{k}=\left(\mathbf{y}_{1, k} \cdots \mathbf{y}_{M, k}\right)$ where $M$ is the number of observations at time step $k$, we deal with these $M$ observations one after another to obtain individual update distributions and their corresponding existence probabilities. The procedure is as follows:

$$
\begin{gathered}
\left\{\begin{array}{l}
f_{i, 0}\left(\mathbf{x}_{i, k}\right) \\
p_{i, k}^{0}
\end{array}\right\}_{i=1}^{N_{k-1}} \stackrel{y_{1, k}}{\rightarrow}\left\{\begin{array}{l}
f_{i, 1}\left(\mathbf{x}_{i, k}\right) \\
p_{i, k}^{1}
\end{array}\right\}_{i=1}^{N_{k-1}} \rightarrow \cdots \stackrel{y_{j-1, k}}{\rightarrow} \\
\left\{\begin{array}{l}
f_{i, j-1}\left(\mathbf{x}_{i, k}\right) \\
p_{i, k}^{-1}
\end{array}\right\}_{i=1}^{N_{k-1}} \stackrel{y_{j, k}}{\rightarrow} \cdots \stackrel{y_{M, k}}{\rightarrow}\left\{\begin{array}{l}
f_{i, M}\left(\mathbf{x}_{i, k}\right) \\
p_{i, k}^{M}
\end{array}\right\}_{i=1}^{N_{k-1}}
\end{gathered}
$$

where $f_{i, 0}\left(\mathbf{x}_{i, k}\right)=f_{i}\left(\mathbf{x}_{i, k} \mid \mathbf{y}_{1: k-1}\right)$ and $p_{i, k}^{0}=p_{i, k \mid k-1}$. After observation $\mathbf{y}_{j-1, k}$ is processed, the update distributions and their corresponding existence probabilities are $f_{i, j-1}$ $\left(\mathbf{x}_{i, k}\right), i=1 \cdots N_{k-1}$ and $p_{i, k}^{j-1}, i=1 \cdots N_{k-1}$, respectively. Using the Bayesian rule to handle observation $\boldsymbol{y}_{j, k}$, we can obtain the update distribution as follows:

$$
f_{i, j}^{u}\left(\mathbf{x}_{i, k}\right)=\frac{p_{D, k} p_{i, k}^{j-1} g_{i}\left(\mathbf{y}_{j, k} \mid \mathbf{x}_{i, k}\right) f_{i, j-1}\left(\mathbf{x}_{i, k}\right)}{\lambda_{c, k}+p_{D, k} \sum_{e=1}^{N_{k-1}} p_{e, k}^{j-1} \int g_{e}\left(\mathbf{y}_{j, k} \mid \mathbf{x}_{e, k}\right) f_{e, j-1}\left(\mathbf{x}_{e, k}\right) d \mathbf{x}_{e, k}}
$$

where $\lambda_{c, k}$ is the clutter density, $p_{D, k}$ is the state-independent detection probability of the target, and $g_{i}\left(\cdot \mid \mathbf{x}_{i, k}\right)$ is the measurement likelihood. Normalizing Equation 6, we obtain the update distribution $f_{i, j}^{a}\left(\mathbf{x}_{i, k}\right)$ as:

$$
f_{i, j}^{a}\left(\mathbf{x}_{i, k}\right)=\frac{1}{p_{i, k}^{a, j}} f_{i, j}^{u}\left(\mathbf{x}_{i, k}\right)
$$

where $p_{i, k}^{a, j}$ is the existence probability of the update distribution $f_{i, j}^{a}\left(\mathbf{x}_{i, k}\right)$, and is defined as:

$$
p_{i, k}^{a, j}=\int f_{i, j}^{u}\left(\mathbf{x}_{i, k}\right) d \mathbf{x}_{i, k}
$$

Given that observation $\boldsymbol{y}_{j, k}$ may originate from one of the targets or from clutter, therefore, we must determine whether observation $\boldsymbol{y}_{j, k}$ originates from target $i$ or not. This process can be accomplished by comparing previous existence probability $p_{i, k}^{j-1}$ with existence probability $p_{i, k}^{a, j}$. If $p_{i, k}^{a, j}>p_{i, j-1}$, then observation $\boldsymbol{y}_{j, k}$ may originate from target $i$; in this case, the update distribution $f_{i, j}\left(\mathbf{x}_{i, k}\right)$ should be $f_{i, j}^{a}\left(\mathbf{x}_{i, k}\right)$. Otherwise, observation $\boldsymbol{y}_{j, k}$ may originate from other targets or from clutter; in this case, previous update distribution $f_{i, j-1}\left(\mathbf{x}_{i, k}\right)$ should be maintained. Therefore, we have:

$$
f_{i, j}\left(\mathbf{x}_{i, k}\right)=\left\{\begin{array}{ll}
f_{i, j}^{a}\left(\mathbf{x}_{i, k}\right) ; & \text { if } p_{i, k}^{a, j}>p_{i, k}^{j-1} \\
f_{i, j-1}\left(\mathbf{x}_{i, k}\right) ; & \text { if } p_{i, k}^{a, j} \leq p_{i, k}^{j-1}
\end{array}, j=1 \cdots M\right.
$$

The existence probability of update distribution $f_{i, j}\left(\mathbf{x}_{i, k}\right)$ can be given by:

$$
p_{i, k}^{j}= \begin{cases}p_{i, k}^{a, j} ; & \text { if } p_{i, k}^{a, j}>p_{i, k}^{j-1} \\ p_{i, k}^{j-1} ; & \text { if } p_{i, k}^{a, j} \leq p_{i, k}^{j-1}, j=1 \cdots M\end{cases}
$$

After all the $M$ observations are processed, we obtain the update distributions $f_{i}\left(\mathbf{x}_{i, k} \mid \mathbf{y}_{1: k}\right), i=1 \cdots N_{k-1}$ at time step $k$ and their corresponding existence probabilities $p_{i, k}, i=1 \cdots N_{k-1}$ as:

$$
\begin{aligned}
& f_{i}\left(\mathbf{x}_{i, k} \mid \mathbf{y}_{1: k}\right)=f_{i, M}\left(\mathbf{x}_{i, k}\right), i=1 \cdots N_{k-1} \\
& p_{i, k}=p_{i, k}^{M}, i=1 \cdots N_{k-1}
\end{aligned}
$$

From Equations 6, 7, 8, 9, 10, 11, and 12, it is clear that $f_{i}\left(\mathbf{x}_{i, k} \mid \mathbf{y}_{1: k}\right)=f_{i}\left(\mathbf{x}_{i, k} \mid \mathbf{y}_{1: k-1}\right)$ and $p_{i, k}=p_{i, k \mid k-1}$ if no measurement is originated from target $i$. This phenomenon indicates that this filter uses the prediction marginal distribution and existence probability of a target as its update marginal distribution and existence probability, respectively, if the target is missing from the incoming measurements and also indicates that this filter has a sufficient memory to missing targets.

Please note that individual targets are not tracked independently in the proposed filter. As shown in Equation 6, 
we use the marginal distributions $f_{e, j-1}\left(\mathbf{x}_{e, k}\right), e=1 \cdots$ $N_{k-1}$ of individual targets to obtain the update distribution $f_{i, j}^{u}\left(\mathbf{x}_{i, k}\right)$ of target $i$. Therefore, individual targets are dependent in this filter.

In addition to existing targets, new targets may appear at time step $k$. Given that SMB filter is measurement driven, the marginal distributions of the new targets should be generated from the observations at time step $k$. To enable the SMB filter to track the new targets appearing at time step $k$, we extend update distributions $f_{i}\left(\mathbf{x}_{i, k} \mid \mathbf{y}_{1: k}\right), i=1 \cdots N_{k-1}$ to include the marginal distributions of the new targets as:

$$
\left\{f_{i}\left(\mathbf{x}_{i, k} \mid \mathbf{y}_{1: k}\right)\right\}_{i=1}^{N_{k}}=\left\{f_{i}\left(\mathbf{x}_{i, k} \mid \mathbf{y}_{1: k}\right)\right\}_{i=1}^{N_{k-1}} \cup\left\{f_{\gamma}^{j}\left(\mathbf{x}_{i, k}\right)\right\}_{j=1}^{M}
$$

The existence probabilities of the extended update distributions are given by:

$$
\left\{p_{i, k}\right\}_{i=1}^{N_{k}}=\left\{p_{i, k}\right\}_{i=1}^{N_{k-1}} \cup\left\{p_{\gamma}^{j}\right\}_{j=1}^{M}
$$

where $N_{k}=N_{k-1}+M ; f_{\gamma}^{j}\left(\mathbf{x}_{i, k}\right), j=1 \cdots M$ are the marginal distributions of the new targets; and $p_{\gamma}^{j}, j=1 \cdots M$ are the existence probabilities of the new targets.

Posterior distributions $f_{i}\left(\mathbf{x}_{i, k} \mid \mathbf{y}_{1}: k\right), i=1 \cdots N_{k}$ in Equation 13 are marginal distributions at time step $k$, and existence probabilities $p_{i, k}, i=\begin{array}{lllll}1 & N_{k} & \text { in }\end{array}$ Equation 14 are existence probabilities of the marginal distributions at time step $k$.

\section{Implementation of the SMB filter for linear Gaussian models}

Similar to the GM-PHD filter in [10], the closed-form solution to the recursion of SMB filter requires the following assumptions:

A4: Each target follows a linear Gaussian dynamic model, and the sensor has a linear Gaussian measurement model, i.e.,

$$
\begin{aligned}
& f_{i}\left(\mathbf{x}_{i, k} \mid \mathbf{x}_{i, k-1}\right)=N\left(\mathbf{x}_{i, k} ; \mathbf{F}_{k-1} \mathbf{x}_{i, k-1}, \mathbf{Q}_{k-1}\right) \\
& g_{i}\left(\mathbf{y}_{j, k} \mid \mathbf{x}_{i, k}\right)=N\left(\mathbf{y}_{j, k} ; \mathbf{H}_{k} \mathbf{x}_{i, k}, \mathbf{R}_{k}\right)
\end{aligned}
$$

where $N(\cdot ; \mathbf{m}, \mathbf{P})$ denotes a Gaussian density with mean $\mathbf{m}$ and covariance $\mathbf{P} ; \mathbf{F}_{k-1}$ is a state transition matrix, and $\mathbf{H}_{k}$ is an observation matrix; and $\mathbf{Q}_{k-1}$ and $\mathbf{R}_{k}$ are process noise and observation noise covariance matrices, respectively.
A5: The new marginal distributions at time step $k$ are Gaussian distribution and are generated from observations at time step $k$ as:

$$
f_{\gamma}^{j}\left(\mathbf{x}_{i, k}\right)=N\left(\mathbf{x}_{i, k} ; \mathbf{m}_{\gamma}^{j}, \mathbf{P}_{\gamma}^{j}\right), j=1 \cdots M
$$

where $\mathbf{P}_{\gamma}^{j}$ is the covariance matrix of new distribution $j$, and $\mathbf{m}_{\gamma}^{j}$ is the mean of new distribution $j$. This mean is generated directly from measurement $\mathbf{y}_{j, k}=\left[\begin{array}{ll}x_{k}^{j} & y_{k}^{j}\end{array}\right]^{T}$ and is given by $\mathbf{m}_{\gamma}^{j}=\left[\begin{array}{llll}x_{k}^{j} & 0 & y_{k}^{j} & 0\end{array}\right]^{T}$. At the same time, we also assume that a small existence probability $p_{\gamma}$ is assigned to each new distribution, i.e.,

$$
p_{\gamma}^{j}=p_{\gamma}, j=1 \cdots M
$$

Any measurement received at time step $k$ may be originated from a new target, an existing target, or clutter. Instead of classifying a measurement as belonging to a new target, we use each measurement to generate a new distribution and assign a small existence probability to each new distribution in the filter recursion.

Based on assumptions A4 and A5, we present a closedform solution to the recursion of the SMB filter for linear Gaussian models. This solution consists of the following prediction and update steps:

Prediction step: Given that the marginal distributions of individual targets at time step $k-1$ are:

$$
\begin{aligned}
f_{i}\left(\mathbf{x}_{i, k-1} \mid \mathbf{y}_{1: k-1}\right) & =N\left(\mathbf{x}_{i, k-1} ; \mathbf{m}_{i, k-1}, \mathbf{P}_{i, k-1}\right), \\
i & =1 \cdots N_{k-1}
\end{aligned}
$$

and that existence probabilities of individual marginal distributions are denoted as $p_{i, k-1}, i=1 \cdots N_{k-1}$. Using Lemmas 1 and 2 in [10], individual prediction distributions can be derived from Equations 4 and 15 and are given by:

$$
\begin{aligned}
f_{i}\left(\mathbf{x}_{i, k} \mid \mathbf{y}_{1: k-1}\right) & =N\left(\mathbf{x}_{i, k} ; \mathbf{m}_{i, k \mid k-1}, \mathbf{P}_{i, k \mid k-1}\right), \\
i & =1 \cdots N_{k-1}
\end{aligned}
$$

where $\mathbf{m}_{i, k \mid k-1}$ and $\mathbf{P}_{i, k \mid k-1}$ are the mean and covariance of prediction distribution $i$, respectively, and are given by:

$$
\begin{aligned}
\mathbf{m}_{i, k \mid k-1} & =\mathbf{F}_{k-1} \mathbf{m}_{i, k-1}, \\
\mathbf{P}_{i, k \mid k-1} & =\mathbf{Q}_{k-1}+\mathbf{F}_{k-1} \mathbf{P}_{i, k-1} \mathbf{F}_{k-1}^{T}
\end{aligned}
$$

where $T$ denotes the transpose. The existence probabilities for the prediction distributions $N\left(\mathbf{x}_{i, k} ; \mathbf{m}_{i, k \mid k}-1\right.$, $\left.\mathbf{P}_{i, k \mid k-1}\right), i=1 \cdots N_{k-1}$ are given by: 


$$
p_{i, k \mid k-1}=p_{S, k}\left(t_{k}, t_{k-1}\right) p_{i, k-1}, i=1 \cdots N_{k-1}
$$

where $p_{S, k}\left(t_{k}, t_{k-1}\right)$ is the time-dependent survival probability of the target and is defined as:

$$
p_{S, k}\left(t_{k,} t_{k-1}\right)=\exp \left(-\frac{t_{k}-t_{k-1}}{\delta \cdot T}\right)
$$

where $t_{k}$ is the time of time step $k, T$ is the sampling period, and $\delta$ is a given parameter. This definition is reasonable because the survival probability of an existing target decreases as time interval increases.

Update step: In this step, we deal with the received observations $\mathrm{y}_{k}=\left(\mathrm{y}_{1, k} \cdots \mathrm{y}_{M, k}\right)$ one after another by using the Bayesian rule to obtain individual update distributions and their corresponding existence probabilities.

Let $f_{i, 0}\left(\mathbf{x}_{i, k}\right)=f_{i}\left(\mathbf{x}_{i, k} \mid \mathbf{y}_{1: k-1}\right)$ and $p_{i, k}^{0}=p_{i, k \mid k-1}$; and let $f_{i, j-1}\left(\mathbf{x}_{i, k}\right)=N\left(\mathbf{x}_{i, k} ; \mathbf{m}_{i, k}^{j-1}, \mathbf{P}_{i, k}^{j-1}\right), i=1 \cdots N_{k-1}$ denote the individual update distributions after observation $\mathbf{y}_{j-1, k}$ is processed. Using Lemmas 1 and 2 in [10], after observation $\mathbf{y}_{j, k}$ is processed, the update distribution $f_{i, j}\left(\mathbf{x}_{i, k}\right)$ and its corresponding existence probability $p_{i, k}^{j}$ are as follows:

$$
\begin{aligned}
& f_{i, j}\left(\mathbf{x}_{i, k}\right)=N\left(\mathbf{x}_{i, k} ; \mathbf{m}_{i, k}^{j}, \mathbf{p}_{i, k}^{j}\right)
\end{aligned}
$$

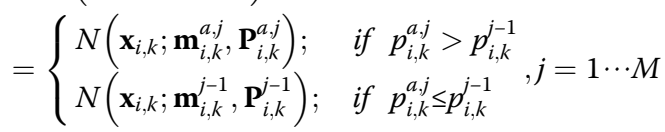

$$
\begin{aligned}
& p_{i, k}^{j}= \begin{cases}p_{i, k}^{a, j} ; & \text { if } p_{i, k}^{a, j}>p_{i, k}^{\mathrm{j}-1} \\
p_{i, k}^{j-1} ; & \text { if } p_{i, k}^{a, j} \leq p_{i, k}^{\mathrm{j}-1}\end{cases}
\end{aligned}
$$

where

$$
\begin{aligned}
& \mathbf{m}_{i, k}^{a, j}=\mathbf{m}_{i, k}^{\mathrm{j}-1}+\mathbf{A}_{i} \cdot\left(\mathbf{y}_{j, k}-\mathbf{H}_{k} \mathbf{m}_{i, k}^{\mathrm{j}-1}\right) \\
& \mathbf{P}_{i, k}^{a, j}=\left(\mathbf{I}-\mathbf{A}_{i} \cdot \mathbf{H}_{k}\right) \mathbf{P}_{i, k}^{j-1} \\
& \mathbf{A}_{i}=\mathbf{P}_{i, k}^{j-1} \mathbf{H}_{k}^{T}\left(\mathbf{H}_{k} \mathbf{P}_{i, k}^{\mathrm{j}-1} \mathbf{H}_{k}^{T}+\mathbf{R}_{k}\right)^{-1} \\
& p_{i, k}^{a, j}=\frac{p_{D, k} p_{i, k}^{j-1} N\left(\mathbf{y}_{j, k} ; \mathbf{H}_{k} \mathbf{m}_{i, k}^{j-1}, \mathbf{H}_{k} \mathbf{P}_{i, k}^{\mathrm{j}-1} \mathbf{H}_{k}^{T}+\mathbf{R}_{k}\right)}{\lambda_{c, k}+p_{D, k} \sum_{e=1}^{N_{k-1}} p_{e, k}^{j-1} N\left(\mathbf{y}_{j, k} ; \mathbf{H}_{k} \mathbf{m}_{e, k}^{j-1}, \mathbf{H}_{k} \mathbf{P}_{e, k}^{\mathrm{j}-1} \mathbf{H}_{k}^{T}+\mathbf{R}_{k}\right)}
\end{aligned}
$$

After all the $M$ observations are processed, we obtain the update distributions $f_{i, M}\left(\mathbf{x}_{i, k}\right)=N\left(\mathbf{x}_{i, k} ; \mathbf{m}_{i, k}^{M}, \mathbf{P}_{i, k}^{M}\right)$, $i=1 \cdots N_{k-1}$ and their existence probabilities $p_{i, k}^{M}, i=1$

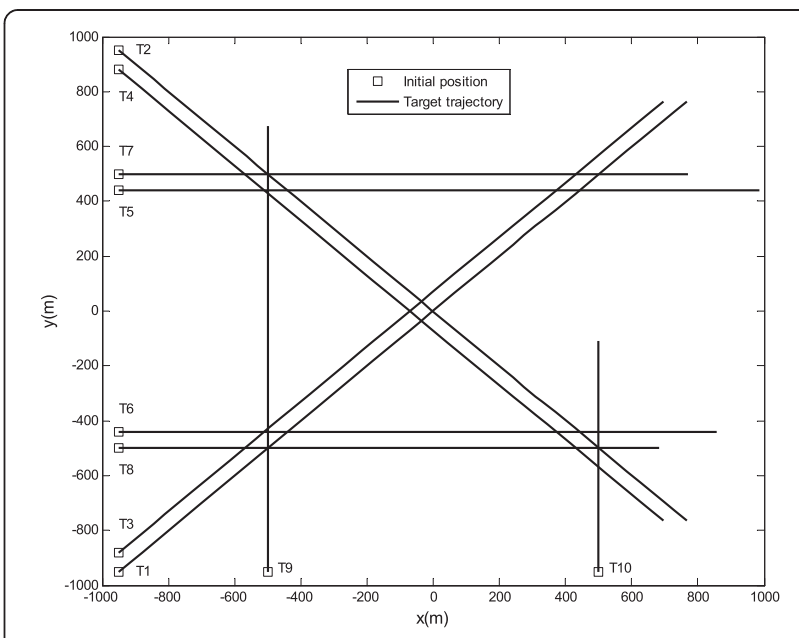

Fig. 1 True target trajectories

$\cdots N_{k-1}$. The update distribution $f_{i}\left(\mathbf{x}_{i, k} \mid \mathbf{y}_{1: k}\right)$ at time step $k$ and their corresponding existence probability $p_{i, k}$ are given by:

$$
\begin{aligned}
f_{i}\left(\mathbf{x}_{i, k} \mid \mathbf{y}_{1: k}\right) & =N\left(\mathbf{x}_{i, k} ; \mathbf{m}_{i, k}, \mathbf{P}_{i, k}\right)=f_{i, M}\left(\mathbf{x}_{i, k}\right) \\
& =N\left(\mathbf{x}_{i, k} ; \mathbf{m}_{i, k}^{M}, \mathbf{P}_{i, k}^{M}\right), i=1 \cdots N_{k-1} \\
p_{i, k}=p_{i, k}^{M}, i & =1 \cdots N_{k-1}
\end{aligned}
$$

To enable the SMB filter to track the new targets appearing at time step $k$, we extend update distributions $f_{i}\left(\mathbf{x}_{i, k} \mid \mathbf{y}_{1: k}\right), i=1 \cdots N_{k-1}$ to include new marginal distribution from Equation 17 as:

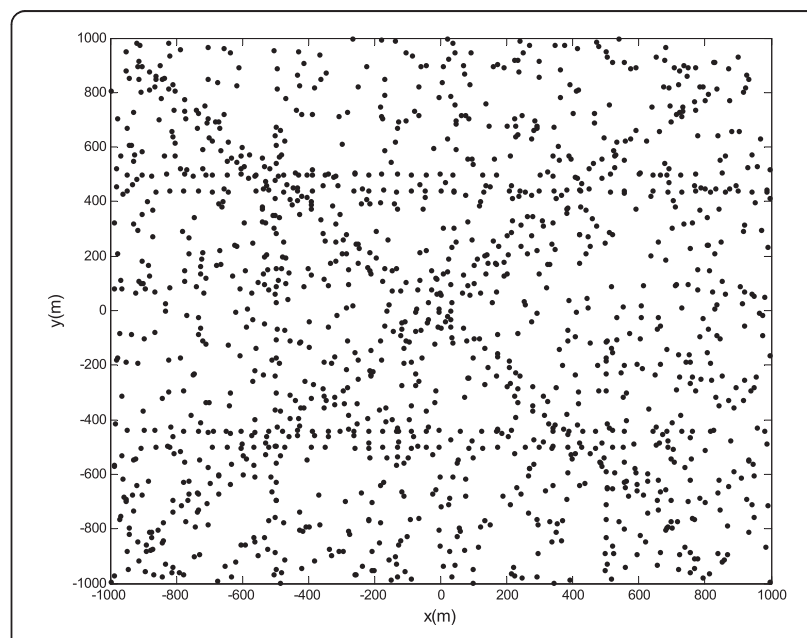

Fig. 2 Measurement data 


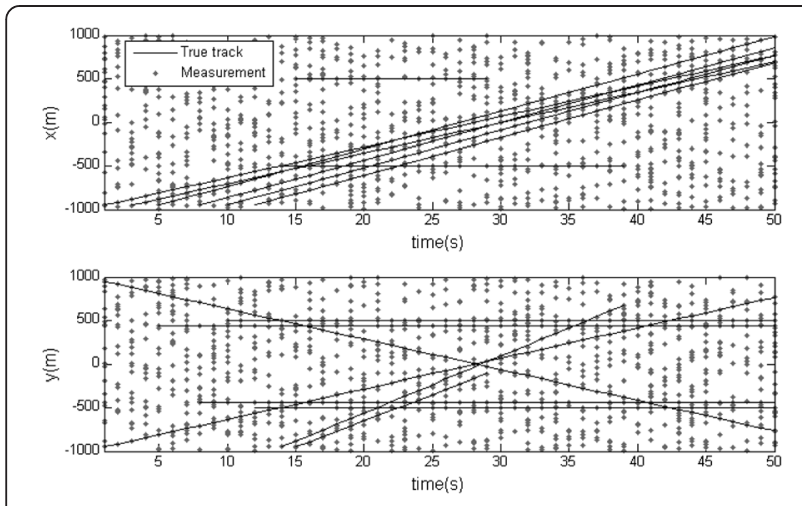

Fig. 3 1-D view of true tracks and measurement data

$$
\begin{aligned}
\left\{f_{i}\left(\mathbf{x}_{i, k} \mid \mathbf{y}_{1: k}\right)\right\}_{i=1}^{N_{k}}= & \left\{N\left(\mathbf{x}_{i, k} ; \mathbf{m}_{i, k}, \mathbf{P}_{i, k}\right)\right\}_{i=1}^{N_{k}} \\
= & \left\{N\left(\mathbf{x}_{i, k} ; \mathbf{m}_{i, k}, \mathbf{P}_{i, k}\right)\right\}_{i=1}^{N_{k-1}} \\
& \cup\left\{N\left(\mathbf{x}_{i, k} ; \mathbf{m}_{\gamma}^{j}, \mathbf{P}_{\gamma}^{j}\right)\right\}_{j=1}^{M}
\end{aligned}
$$

The existence probabilities of the extended update distributions are given by:

$$
\left\{p_{i, k}\right\}_{i=1}^{N_{k}}=\left\{p_{i, k}\right\}_{i=1}^{N_{k-1}} \cup\left\{p_{\gamma}^{j}\right\}_{j=1}^{M}
$$

An implementation of the proposed filter for linear Gaussian models comprises, in addition to the above prediction and update steps, the following multi-target state extraction step:

Multi-target state extraction step: Given the extended update distributions $f_{i}\left(\mathrm{x}_{i, k} \mid \mathrm{y}_{1: k}\right)=N\left(\mathrm{x}_{i, k} ; \mathrm{m}_{i, k}, \mathrm{P}_{i, k}\right), i=1$ $\cdots N_{k}$ and their existence probabilities $p_{i, k}, i=1 \cdots N_{k}$, we first eliminate the Gaussian distribution whose

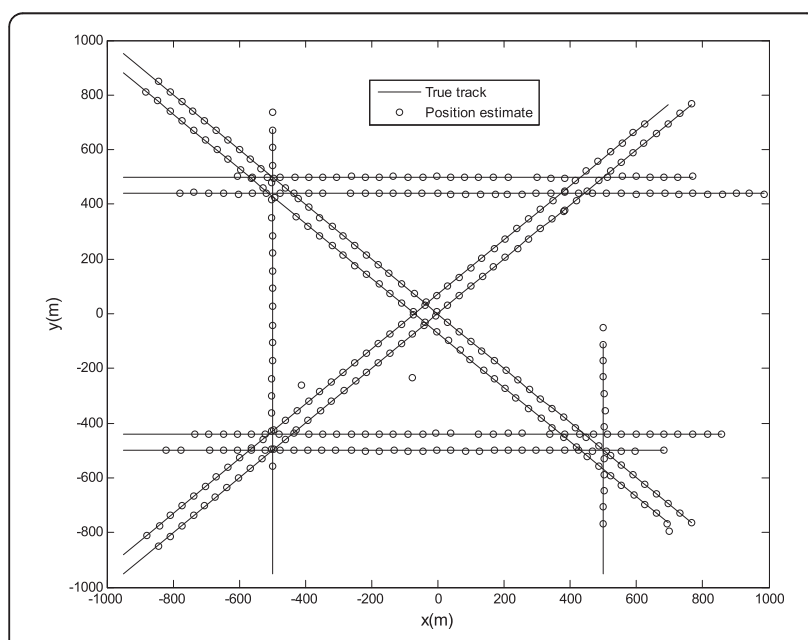

Fig. 4 Tracking result of the SMB filter

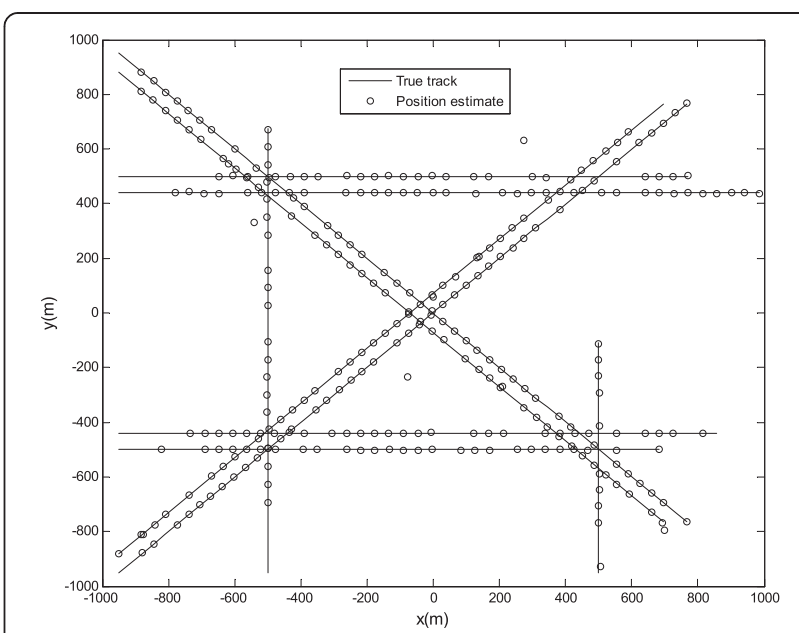

Fig. 5 Tracking result of the PHD-M filter

existence probability is smaller than a given threshold $\tau$. After pruning, the remaining Gaussian distributions and their existence probabilities are used as inputs for the next filtering recursion. We then select the Gaussian distributions with existence probabilities $p_{i, k}>0.5$ as outputs of the filter. The mean of a selected Gaussian distribution is the state estimate of a target.

\section{Simulation results}

We select the PHD/CPHD filters with measurementdriven birth intensity [19] as two contesting comparison objects in the simulation. The selected filters adapt the target birth intensity at each processing step with the use of the received measurements, thereby eliminating the need for prior specification of birth intensities [19].

We consider an example in this section. This example shows the tracking performances of the PHD filter, the

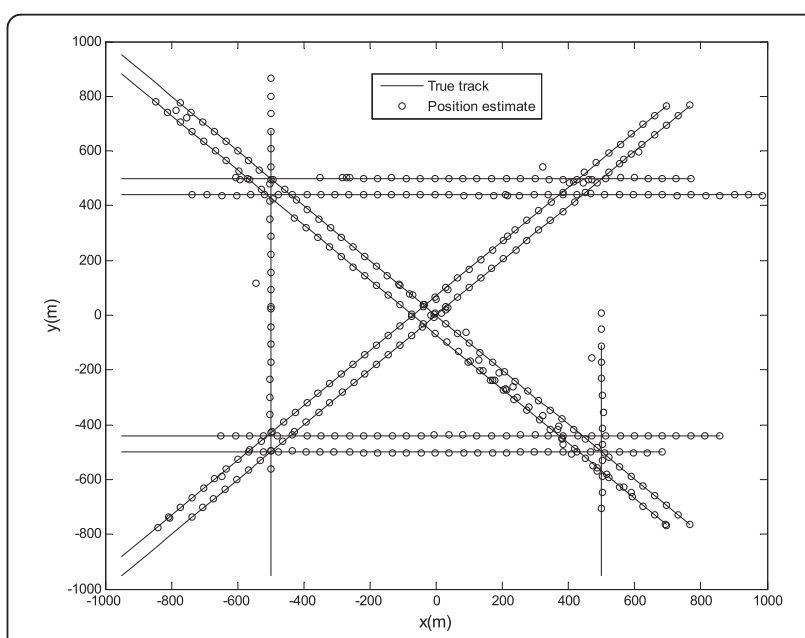

Fig. 6 Tracking result of the CPHD-M filter 


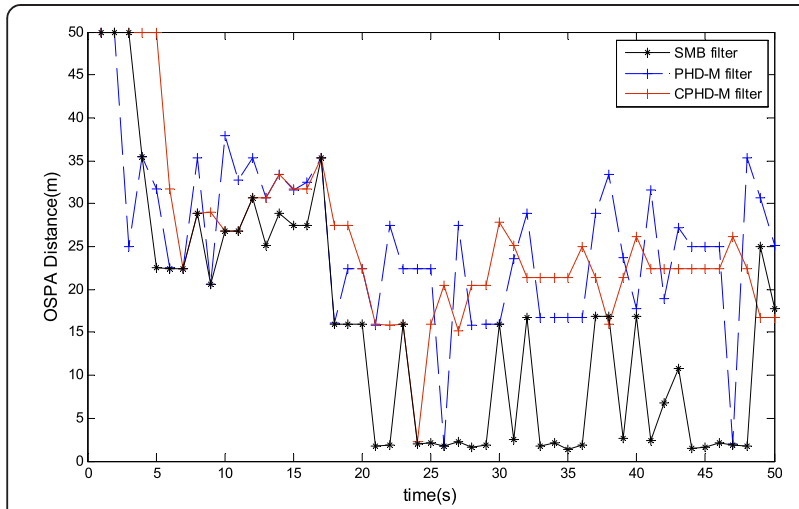

Fig. 7 OSPA distances of the three filters

CPHD filter, and the proposed SMB filter for linear Gaussian models. In this example, the pruning procedure presented in [10] is performed at each time step of both GM-PHD filter and GM-CPHD filter. The state extraction in GM-PHD filter involves selecting the means of the Gaussians that have weights greater than 0.5 as the state estimates [10], whereas the state extraction in GM-CPHD filter involves estimating the number of targets and then extracting the corresponding number of Gaussians with the highest weights as state estimates [22]. To assess the tracking performances of these three filters, we use the OSPA distance [25] as the measure, where parameters are set as $p=2$ and $c=50 \mathrm{~m}$.

Example: Targets move at a constant velocity in a 2-D region $[-1,000(\mathrm{~m}), 1,000(\mathrm{~m})] \times[-1,000(\mathrm{~m}), 1,000(\mathrm{~m})]$ in this example. The state vector is represented by $\mathbf{x}_{i, k}=$ $\left[\begin{array}{llll}x_{i, k} & \dot{x}_{i, k} & y_{i, k} & \dot{y}_{i, k}\end{array}\right]^{T}$, and the state transition matrix $\mathbf{F}_{k-1}$ and covariance matrix $\mathbf{Q}_{k-1}$ in Equation 15 are given by:

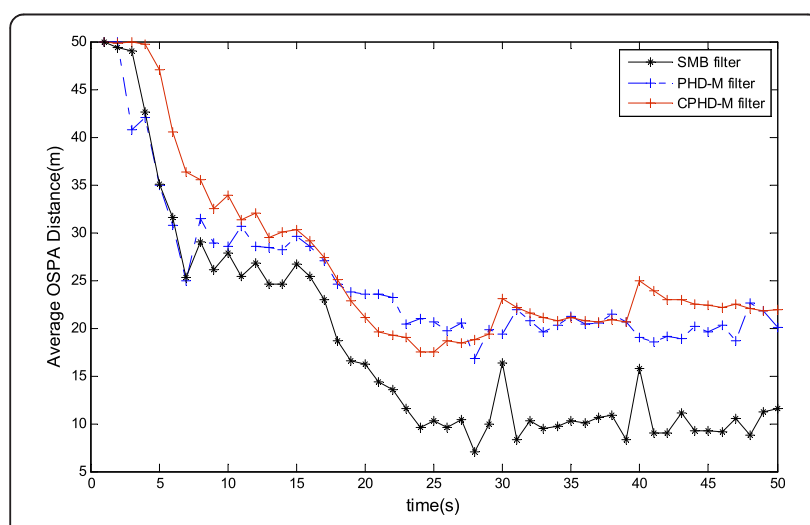

Fig. 8 Average OSPA distances of the three filters

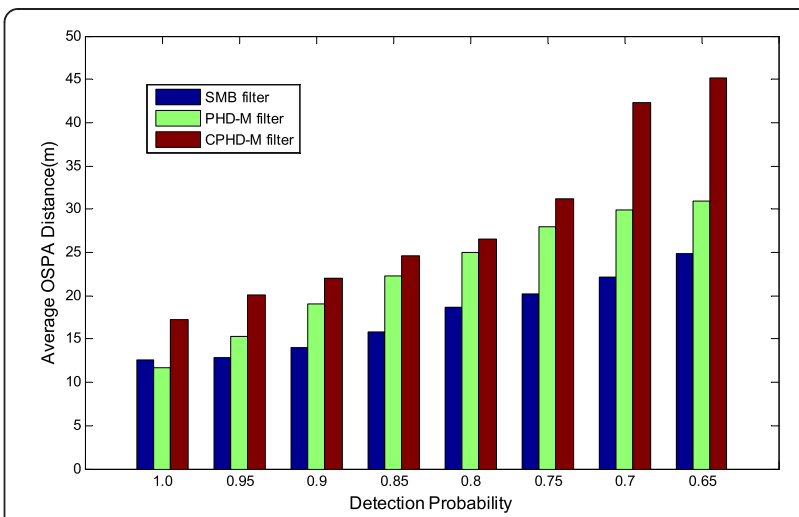

Fig. 9 Average OSPA distances of the three filters for different detection probabilities

$$
\begin{aligned}
\mathbf{F}_{k-1} & =\left[\begin{array}{cccc}
1 & \Delta t_{k} & 0 & 0 \\
0 & 1 & 0 & 0 \\
0 & 0 & 1 & \Delta t_{k} \\
0 & 0 & 0 & 1
\end{array}\right], \\
\mathbf{Q}_{k-1} & =\left[\begin{array}{cc}
\Delta t_{k}^{2} / 2 & 0 \\
\Delta t_{k} & 0 \\
0 & \Delta t_{k}^{2} / 2 \\
0 & \Delta t_{k}
\end{array}\right]\left[\begin{array}{cccc}
\Delta t_{k}^{2} / 2 & \Delta t_{k} & 0 & 0 \\
0 & 0 & \Delta t_{k}^{2} / 2 & \Delta t_{k}
\end{array}\right] \sigma_{v}^{2}
\end{aligned}
$$

where $\Delta t_{k}=t_{k}-t_{k-1}$ and $\sigma_{v}$ is the standard deviation of the process noise. The observation matrix $\mathbf{H}_{k}$ and covariance matrix $\mathbf{R}_{k}$ in Equation 16 are:

$$
\mathbf{H}_{k}=\left[\begin{array}{llll}
1 & 0 & 0 & 0 \\
0 & 0 & 1 & 0
\end{array}\right], \mathbf{R}_{k}=\left[\begin{array}{ll}
1 & 0 \\
0 & 1
\end{array}\right] \sigma_{w}^{2}
$$

where $\sigma_{w}$ is the standard deviation of the observation noise.

Ten targets exist in this example. Targets 1 to 8 appear at $t=1 \mathrm{~s}, t=1 \mathrm{~s}, t=3 \mathrm{~s}, t=3 \mathrm{~s}, t=5 \mathrm{~s}, t=8 \mathrm{~s}$, $t=10 \mathrm{~s}$, and $t=12 \mathrm{~s}$, respectively, and continue to exist in the remaining time. Targets 9 and 10 appear at $t=14 \mathrm{~s}$ and $t=15 \mathrm{~s}$ but disappear at $t=40 \mathrm{~s}$ and $t=30 \mathrm{~s}$, respectively. The initial states of the individual targets are as follows:

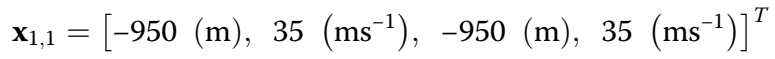

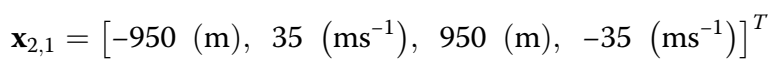

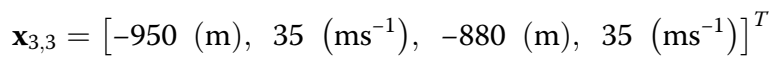

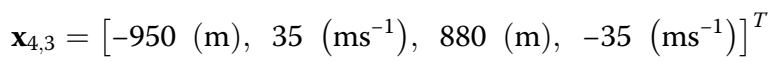

Table 1 Average performing time (s) of a Monte Carlo run for different detection probabilities

\begin{tabular}{lllllllll}
\hline$P_{D, k}$ & 1.0 & 0.95 & 0.9 & 0.85 & 0.8 & 0.75 & 0.7 & 0.65 \\
\hline SMB filter & 23.21 & 22.44 & 22.02 & 21.73 & 21.36 & 20.88 & 20.35 & 19.75 \\
PHD-M filter & 1.84 & 2.46 & 3.09 & 4.09 & 4.29 & 4.98 & 6.25 & 6.50 \\
CPHD-M filter & 28.13 & 29.39 & 29.75 & 29.87 & 29.07 & 28.71 & 28.27 & 27.91 \\
\hline
\end{tabular}




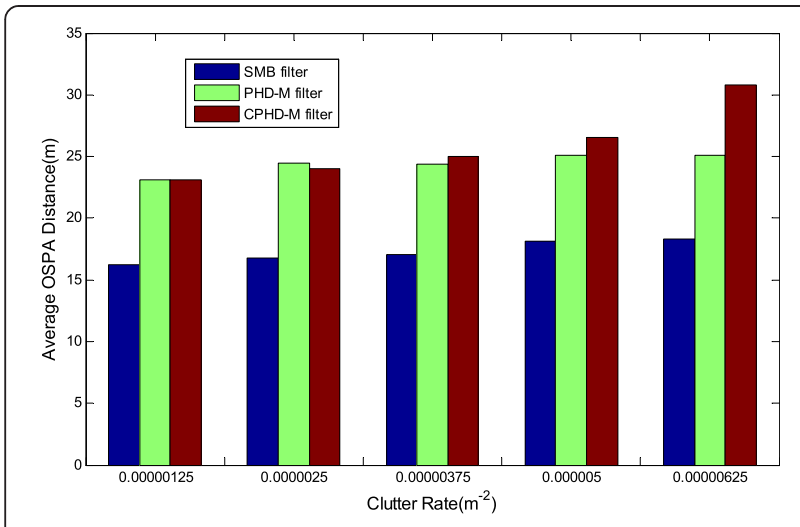

Fig. 10 Average OSPA distances of the three filters for different clutter rates

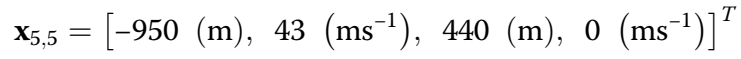

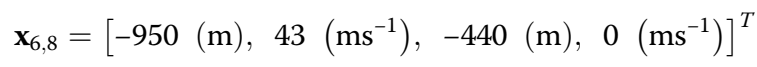

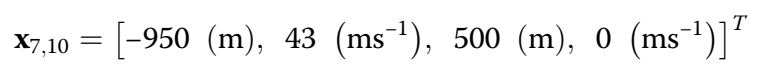

$$
\begin{aligned}
& \mathbf{x}_{8,12}=\left[\begin{array}{llll}
-950(\mathrm{~m}), 43\left(\mathrm{~ms}^{-1}\right), & -500(\mathrm{~m}), & 0 & \left.\left(\mathrm{~ms}^{-1}\right)\right]^{T}
\end{array}\right. \\
& \mathbf{x}_{9,14}=\left[\begin{array}{llll}
-500(\mathrm{~m}), & 0\left(\mathrm{~ms}^{-1}\right), & -950(\mathrm{~m}), & \left.65\left(\mathrm{~ms}^{-1}\right)\right]^{T}
\end{array}\right. \\
& \mathbf{x}_{10,15}=\left[\begin{array}{llll}
-500(\mathrm{~m}), & 0 & \left(\mathrm{~ms}^{-1}\right), & \left.-950(\mathrm{~m}), 60\left(\mathrm{~ms}^{-1}\right)\right]^{T}
\end{array}\right.
\end{aligned}
$$

The true trajectories of the 10 targets are shown in Fig. 1.

The sampling period of the sensor is $T=1 \mathrm{~s}$, and the measurements are generated by using $p_{S, k}=1.0, p_{D, k}=0.8$, $\sigma_{v}=1 \mathrm{~ms}^{-2}, \sigma_{w}=2 \mathrm{~m}$, and $\lambda_{c, k}=5 \times 10^{-6} \mathrm{~m}^{-2}$. Figure 2 shows the measurement data in the horizontal plane. A $1-\mathrm{D}$ view of these measurements along with the true tracks is shown in Fig. 3.

To detect the targets in the surveillance space and to estimate their states from the measurement data, we set the corresponding parameters of these three filters to $\lambda_{c, k}=5 \times 10^{-6} \mathrm{~m}^{-2}, p_{D, k}=0.8, \sigma_{v}=1 \mathrm{~ms}^{-2}, \sigma_{w}=2 \mathrm{~m}, \tau=$ $10^{-3}$, and $\delta=2$. The weight $w_{\gamma}^{j}$ of the new distribution in Gaussian mixture PHD/CPHD filters and the existence probability $p_{\gamma}^{j}$ of the new distribution in the SMB filter are set to $w_{\gamma}^{j}=p_{\gamma}^{j}=0.05$. The covariance of the new distribution is $\mathbf{P}_{\gamma}^{j}=\left(\operatorname{diag}\left(\left[\begin{array}{llll}50 & 25 & 50 & 25\end{array}\right]\right)\right)^{2}$. In both PHD and CPHD filters, the survival probability $p_{S, k}$ is set to $p_{S, k}=1.0$.
To deal with the measurement data, we use SMD filter, Gaussian mixture PHD filter with a measurementdriven birth intensity (PHD-M filter), and Gaussian mixture CPHD filter with the measurement-driven birth intensity (CPHD-M filter). Figures 4, 5, and 6 respectively show the results given by these three filters.

Figures 4, 5, and 6 shows that these three filters can detect multiple targets and estimate their states in the presence of clutter as well as target appearance and disappearance. These three filters need a few time steps to confirm the appearance of new targets. Given the fast response of the SMB and PHD filters to the target appearance, they take fewer time steps than the CPHD filter in detecting a new target. The PHD filter fails to provide the state estimates of existing targets if these targets are missing from the incoming measurements owing to the poor memory of the filter. Meanwhile, the SMB and CPHD filters can provide the state estimates of missed targets because they have a sufficient memory for the existing targets. The sufficient memory also enables the CPHD filter and PHD filter to give state estimates of a disappearing target at the disappearing time, whereas the PHD filter does not provide the state estimate of a disappearing target because of its fast response to target disappearance. The strong memory of the CPHD filter causes it to provide excessive state estimates of existing and disappearing targets. Figure 7 shows the OSPA distances of these three filters and illustrates that the SMB filter performs best in terms of OSPA distance.

We run 100 Monte Carlo trials for each filter to obtain the average OSPA distance. The result shown in Fig. 8 reveals the following: 1) the SMB filter is the best at detecting and tracking multiple targets because the average OSPA distance of this filter is the smallest; 2) the average OSPA distances of SMB filter and PHD filter decrease faster than that of the CPHD filter between times $t=1 \mathrm{~s}$ and $t=15 \mathrm{~s}$ because they respond faster to the target appearance; and 3) each time a target disappears, a peak emerges in the plots of both the SMB and CPHD filters. This condition occurs because these two filters provide the state estimate of a disappearing target and are penalized with the cutoff distance, $c=50 \mathrm{~m}$, at the time of the target's disappearance.

Figure 9 shows the average OSPA distances of these three filters for different detection probabilities with clutter rate fixed at $\lambda_{c, k}=5 \times 10^{-6} \mathrm{~m}^{-2}$. The average OSPA distance increases as the detection probability

Table 2 Average performing time (s) of a Monte Carlo run for different clutter rates

\begin{tabular}{llllll}
\hline$\lambda_{c, k}\left(\mathrm{~m}^{-2}\right)$ & 0.00000125 & 0.0000025 & 0.00000375 & 0.000005 & 0.00000625 \\
\hline SMB filter & 7.90 & 12.37 & 16.65 & 21.36 & 25.78 \\
PHD-M filter & 0.79 & 1.48 & 2.37 & 4.29 & 7.03 \\
CPHD-M filter & 14.48 & 19.89 & 24.16 & 29.07 & 33.22 \\
\hline
\end{tabular}


decreases. In addition, the average OSPA distance of the SMB filter is the smallest for each detection probability smaller than 1.0.

Table 1 shows the average performing time of a Monte Carlo run for the different detection probabilities given a fixed clutter rate of $\lambda_{c, k}=5 \times 10^{-6} \mathrm{~m}^{-2}$. Based on Table 1 , the average performing time of the SMB filter is larger than that of the PHD filter but smaller than that of the CPHD filter at each detection probability.

Figure 10 shows the average OSPA distances of the three filters on the basis of different clutter rates with detection probability fixed at $p_{D, k}=0.8$. The average OSPA distance increases as the clutter rate increases. The average OSPA distance of the SMB filter is consistently the smallest at each clutter rate.

Table 2 shows the average performing time of a Monte Carlo run for different clutter rates with a fixed detection probability of $p_{D, k}=0.8$. Based on Table 2 , the average performing time of the SMB filter is larger than that of the PHD filter but is smaller than that of the CPHD filter at each clutter rate.

\section{Conclusions}

In this study, we propose a sequential measurementdriven multi-target Bayesian filter. This filter propagates marginal distributions and existence probabilities for each target. We also present an implementation of the proposed filter for linear Gaussian models. The proposed filter can process new observations as soon as these new observations become available, thereby reducing data processing delay. This filter can track multiple targets in the presence of clutter as well as target appearance and disappearance and is applicable to cases in which the detection probability of the target is low and the sampling period is long. Simulation results show that this filter tracks multiple targets better than the PHD and CPHD filters given a low detection probability of the target.

\section{Competing interests}

The authors declare that they have no competing interests.

\section{Acknowledgements}

This work was supported by the National Natural Science Foundation of China (Nos. 61271107 and 61301074), Shenzhen Basic Research Project (No. JCYJ20140418095735618), and National Defense Pre-research Foundation (No. 9140C800501140C80340).

Received: 16 January 2015 Accepted: 23 April 2015

Published online: 15 May 2015

\section{References}

1. J Vermaak, SJ Godsill, P Perez, Monte Carlo filtering for multi-target tracking and data association. IEEE Trans. Aerosp. Electron. Syst. 41(1), 309-31 (2005)

2. R Mahler, Statistical multisource-multitarget information fusion (Artech House, Norwood, MA, 2007), pp. 565-653

3. R Mahler, Multitarget bayes filtering via first-order multitarget moments. IEEE Trans. Aerosp. Electron. Syst. 39(4), 1152-78 (2003)

4. L Lin, Y Bar-Shalom, T Kirubarajan, Tracking labeling and PHD filter for multitarget tracking. IEEE Trans. Aerosp. Electron. Syst. 42(3), 778-95 (2006)
5. JH Yoon, DY Kim, KJ Yoon, Efficient importance sampling function design for sequential Monte Carlo PHD filter. Signal Process. 92, 2315-21 (2012)

6. $\lrcorner$ Yang, HB Ji, A novel track maintenance algorithm for PHD/CPHD filter. Signal Process. 92, 2371-80 (2012)

7. C Ouyang, HB Ji, ZQ Guo, Extensions of the SMC-PHD for jump Markov systems. Signal Process. 92, 1422-30 (2012)

8. BN Vo, SS Doucet, Sequential Monte Carlo methods for multi-target filtering with random finite sets. IEEE Trans. Aerosp. Electron. Syst. 41(4), 1224-45 (2005)

9. HJ Zhang, ZL Jing, SQ Hu, Localization of multiple emitter based on the sequential PHD filter. Signal Process. 90, 34-43 (2010)

10. BN Vo, WK Ma, The Gaussian mixture probability hypothesis density filter. IEEE Trans. Signal Process. 54(11), 4091-104 (2006)

11. K Panta, DE Clark, BN Vo, Data association and management for the Gaussian mixture probability hypothesis. IEEE Trans. Aerosp. Electron. Syst. 45(3), 1003-16 (2009)

12. SA Pasha, BN Vo, HD Tuan, WK Ma, A Gaussian mixture PHD filter for jump Markov system models. IEEE Trans. Aerosp. Electron. Syst. 45(3), 919-36 (2009)

13. ZX Liu, WX Xie, P Wang, Y Yu, A sequential GM-based PHD filter for a linear Gaussian system. Sci. China. Inf. Sci. 56(10), 102302:1-102302:10 (2013)

14. TM Wood, Interacting methods for manoeuvre handling in the GM-PHD filter. IEEE Trans. Aerosp. Electron. Syst. 47(4), 3021-5 (2011)

15. WL Li, YM Jia, Gaussian mixture PHD filter for jump Markov models based on best-fitting Gaussian approximation. Signal Process. 91, 1036-42 (2011)

16. Y Wang, ZL Jing, SQ Hu, JJ Wu, Detection-guided multi-target Bayesian filter. Signal Process. 92, 564-74 (2012)

17. ZX Liu, WX Xie, P Wang, Y Yu, A Gaussian mixture PHD filter with the capability of information hold. Acta. Electronica. Sinica 41(8), 1603-8 (2013)

18. JH Yoon, DY Kin, SH Bae, V Shin, Joint initialization and tracking of multiple moving objects using Doppler information. IEEE Trans. Signal Process. 59(7), 3447-52 (2011)

19. B Ristic, D Clark, BN Vo, BT Vo, Adaptive target birth intensity for PHD and CPHD filter. IEEE Trans. Aerosp. Electron. Syst. 48(2), 1656-68 (2012)

20. K Panta, BN Vo, S Singh, Novel data association schemes for the probability hypothesis density filter. IEEE Trans. Aerosp. Electron. Syst. 43(2), 556-70 (2007)

21. K Panta, DE Clark, BN Vo, Data association and track management for the Gaussian mixture probability hypothesis density filter. IEEE Trans. Aerosp. Electron. Syst. 45(3), 1003-16 (2009)

22. BT Vo, BN Vo, A Cantoni, Analytic implementations of the cardinalized probability hypothesis density filter. IEEE Trans. Signal Process. 55(7), 3553-67 (2007)

23. R Mahler, BT Vo, BN Vo, CPHD filtering with unknown clutter rate and detection profile. IEEE Trans. Signal Process. 59(8), 3497-513 (2011)

24. F Lian, CZ Han, WF Liu, Estimating unknown clutter intensity for PHD filter. IEEE Trans. Aerosp. Electron. Syst. 46(4), 2066-78 (2010)

25. D Schuhmacher, BT Vo, BN Vo, A consistent metric for performance evaluation of multi-object filters. IEEE Trans. Signal Process. 56(8), 3447-57 (2008)

\section{Submit your manuscript to a SpringerOpen ${ }^{\circ}$ journal and benefit from:}

- Convenient online submission

- Rigorous peer review

- Immediate publication on acceptance

- Open access: articles freely available online

- High visibility within the field

- Retaining the copyright to your article

Submit your next manuscript at springeropen.com 